\title{
The exceptionally extended flaring activity in the X-ray afterglow of GRB 050730 observed with Swift and XMM-Newton
}

\author{
M. Perri ${ }^{1}$, D. Guetta ${ }^{2}$, L. A. Antonelli ${ }^{1,2}$, A. Cucchiara ${ }^{3}$, V. Mangano ${ }^{4}$, J. Reeves ${ }^{5}$, L. Angelini ${ }^{5}$, A. P. Beardmore ${ }^{6}$, \\ P. Boyd ${ }^{5}$, D. N. Burrows ${ }^{3}$, S. Campana ${ }^{7}$, M. Capalbi ${ }^{1}$, G. Chincarini ${ }^{7}, 8$, G. Cusumano ${ }^{4}$, P. Giommi ${ }^{1,9}$, J. E. Hill ${ }^{5}$, \\ S. T. Holland ${ }^{5,10}$, V. La Parola ${ }^{4}$, T. Mineo ${ }^{4}$, A. Moretti ${ }^{7}$, J. A. Nousek ${ }^{3}$, J. P. Osborne ${ }^{6}$, C. Pagani ${ }^{3}$, P. Romano ${ }^{7}$, \\ P. W. A. Roming ${ }^{3}$, R. L. C. Starling ${ }^{6}$, G. Tagliaferri ${ }^{7}$, E. Troja ${ }^{4,6}$, L. Vetere ${ }^{1,3}$, and N. Gehrels ${ }^{5}$
}

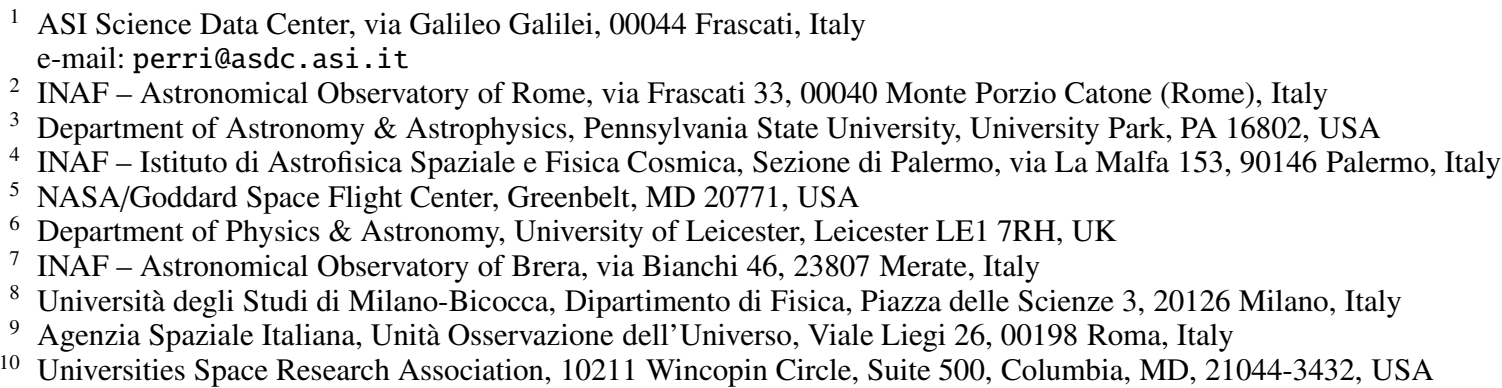

Received 11 August 2006 / Accepted 27 March 2007

ABSTRACT

\begin{abstract}
Aims. We observed the high redshift $(z=3.969)$ GRB 050730 with Swift and XMM-Newton to study its prompt and afterglow emission.

Methods. We carried out a detailed spectral and temporal analysis of Swift and XMM-Newton observations.

Results. The X-ray afterglow of GRB 050730 was found to decline along with time with superimposed intense flaring activity that extended over more than two orders of magnitude in time. Seven distinct re-brightening events starting from $236 \mathrm{~s}$ and up to $41.2 \mathrm{ks}$ after the burst were observed. The underlying decay of the afterglow was well described by a double broken power-law model with breaks at $t_{1}=237 \pm 20 \mathrm{~s}$ and $t_{2}=10.1_{-2.2}^{+4.6} \mathrm{ks}$. The temporal decay slopes before, between and after these breaks were $\alpha_{1}=2.1 \pm 0.3$, $\alpha_{2}=0.44_{-0.08}^{+0.14}$ and $\alpha_{3}=2.40_{-0.07}^{+0.09}$, respectively. The spectrum of the X-ray afterglow was well described by a photoelectrically absorbed power-law with an absorbing column density $N_{\mathrm{H}}^{z}=\left(1.28_{-0.25}^{+0.26}\right) \times 10^{22} \mathrm{~cm}^{-2}$ in the host galaxy. Evidence was found of flaring activity in the early UVOT optical afterglow, simultaneous with that observed in the X-ray band. Strong X-ray spectral evolution during the flaring activity was present. The rise and decay power-law slopes of the first three flares were in the range $0.8-1.8$ using as zero times the beginning and the peak of the flares, respectively. In the majority of flares $(6 / 7)$, the ratio $\Delta t / t_{\mathrm{p}}$ between the duration of the event and the time when the flare peaks was nearly constant and $\sim 0.6-0.7$. We showed that the observed spectral and temporal properties of the first three flares are consistent with being due both to high-latitude emission, as expected if the flares were produced by late internal shocks, and to refreshed shocks, i.e. late time energy injections into the main afterglow shock by slow moving shells ejected from the central engine during the prompt phase. The event fully satisfies the $E_{\mathrm{p}}-E_{\text {iso }}$ Amati relation but is not consistent with the $E_{\mathrm{p}}$ vs. $E_{\text {jet }}$ Ghirlanda relation.
\end{abstract}

Key words. gamma rays: bursts - X-rays: individuals: GRB 050730

\section{Introduction}

The successful launch on 2004 November 20 of the Swift Gamma-ray Burst Explorer (Gehrels et al. 2004) has opened a new era in the study of Gamma Ray Bursts (GRBs). The autonomous and rapid slewing capabilities of Swift allow the prompt (1-2 min) observation of GRBs, discovered and localised by the wide-field gamma-ray (15-350 keV) Burst Alert Telescope (BAT, Barthelmy et al. 2005a), with the two co-aligned narrow-field instruments on-board the observatory: the X-ray Telescope (XRT, Burrows et al. 2005a), operating in the $0.2-10 \mathrm{keV}$ energy band, and the Ultraviolet/Optical Telescope (UVOT, Roming et al. 2005), sensitive in the 1700$6000 \AA$ band. The Swift unique fast pointing capability is crucial in the X-ray energy band, where the reaction times of other satellites are limited to time scales of several hours. With Swift, thanks also to the XRT high sensitivity, for the first it is possible time to study in detail the evolution of GRB X-ray afterglows during their very early phases.

Indeed, one of the main results of Swift is the identification of unexpected and complex features in the early X-ray afterglows. In particular, three distinct phases are observed in the majority of GRB light curves: an early $(t<500 \mathrm{~s}$ from the trigger) steep decline, with a power-law index of $\sim 3$, a second $(t<10 \mathrm{ks})$ very shallow phase with a slope of $\sim 0.5$, a third phase characterized by a more conventional decay slope of $\sim 1$ (e.g. Tagliaferri et al. 2005; Cusumano et al. 2006a; Nousek et al. 2006; O'Brien et al. 2006). In a few cases 
(GRB 050315, Vaughan et al. 2006; GRB 050318, Perri et al. 2005; GRB 050505, Hurkett et al. 2006; GRB 050525A, Blustin et al. 2006; GRB 060614, Mangano et al. 2007) a further steepening with a decay slope of $\sim 2$, consistent with a jet break, is observed. Moreover, Swift had detected in about one-half of the bursts, strong flaring activity in the X-ray energy band superimposed on the afterglow decay (e.g. Burrows et al. 2005b; Romano et al. 2006; Falcone et al. 2006).

The understanding of the origin of these bright X-ray flares is intensively discussed in the literature. A mechanism proposed as responsible for the flaring activity is late internal shocks (Burrows et al. 2005b; Fan \& Wei 2005; Zhang et al. 2006; Liang et al. 2006). In this scenario, the X-ray flares are produced by the same internal dissipation processes which cause the prompt emission, likely internal shocks within the expanding fireball occurring before it is decelerated in the external medium (e.g. Rees \& Mészáros 1994). This model requires that the GRB central engine is still active after the end of the prompt emission and various mechanisms providing such extended internal activity have been put forward (e.g. King et al. 2005; Perna et al. 2006). An alternative scenario has been recently considered by Guetta et al. (2007) who, based on a detailed analysis of the X-ray flaring activity observed in the afterglow of GRB 050713A, interpreted the X-ray flares as due to refreshed shocks, i.e. late time collisions with the main afterglow shock of slow-moving shells ejected from the central engine during the prompt phase (Rees \& Mészáros 1998; Kumar \& Piran 2000; Sari \& Mészáros 2000).

In this paper we present a detailed analysis of Swift and XMM-Newton observations of GRB 050730, focusing on the intense and extended X-ray flaring activity that characterizes its afterglow. In Sect. 2 the observations and the data reduction are presented, in Sect. 3 we describe the temporal analysis and Sect. 4 is dedicated to the spectral analysis. The results are discussed in Sect. 5 and finally in Sect. 6 we summarize our findings. Throughout this paper errors are quoted at the $90 \%$ confidence level for one parameter of interest $\left(\Delta \chi^{2}=2.71\right)$ unless otherwise specified. We adopted the standard $\Lambda$ CDM cosmological parameters of $\Omega_{\mathrm{m}}=0.27, \Omega_{\Lambda}=0.73$ and $H_{0}=70 \mathrm{~km} \mathrm{~s}^{-1} \mathrm{Mpc}^{-1}$. Times are referenced from the BAT trigger $T_{0}$ while temporal and spectral indices are written following the notation $F(t, v) \propto t^{-\alpha} v^{-\beta}$. Results on the optical spectrum of the afterglow of this GRB are reported by Starling et al. (2005), Chen et al. (2005a) and Prochaska et al. (2006). Multi-wavelength observations of the afterglow of GRB 050730 are presented by Pandey et al. (2006).

\section{Observations and data reduction}

\subsection{Swift BAT}

The BAT detected and located GRB 050730 at $T_{0}=$ 19:58:23 UT on 2005 July 30 (Holland et al. 2005). On the basis of the refined ground analysis (Markwardt et al. 2005), the BAT position is $\operatorname{RA}(J 2000)=212^{\circ} .063, \operatorname{Dec}(J 2000)=-3^{\circ} .740$, with a $90 \%$ containment radius of $3^{\prime}$. The BAT prompt emission light curve (Fig. 1) is characterized by a duration of $T_{90}=155 \pm 20 \mathrm{~s}$. The emission starts $\sim 60 \mathrm{~s}$ before the trigger, peaks at $\sim 10 \mathrm{~s}$ after the trigger and declines out to $\sim 180 \mathrm{~s}$ after the trigger.

The time averaged (over $T_{90}$ ) spectrum in the $15-150 \mathrm{keV}$ energy band is well described by a power-law model with an energy index $\beta_{\mathrm{BAT}}=0.5 \pm 0.1$ and $\chi_{r}^{2}=0.71$ (with 56 degrees of freedom, d.o.f.). The total fluence in the $15-150 \mathrm{keV}$ band is $(2.4 \pm 0.3) \times 10^{-6} \mathrm{erg} \mathrm{cm} \mathrm{cm}^{-2}$. Assuming redshift $z=3.969$

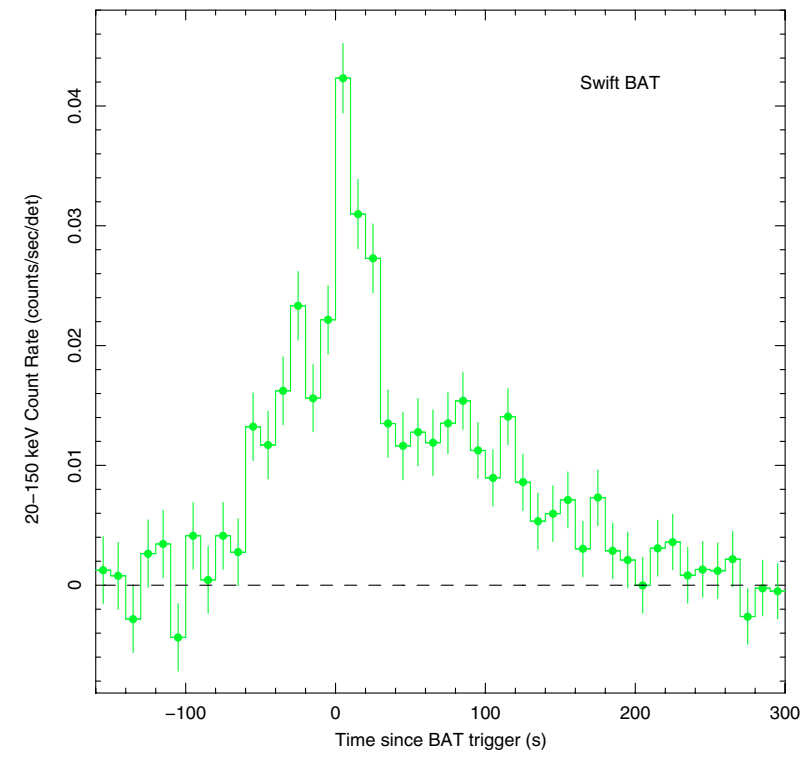

Fig. 1. BAT 20-150 keV background subtracted light curve of the prompt emission of GRB 050730. Data are binned to $10 \mathrm{~s}$ resolution and errors are at the $1 \sigma$ level. The horizontal dashed line indicates the 0 level.

(Chen et al. 2005b, see Sect. 2.2), the isotropic-equivalent radiated energy in the BAT bandpass $(74.5-745.4 \mathrm{keV}$ in the burst rest frame $)$ is $E_{\text {iso }}^{\mathrm{BAT}}=(8.0 \pm 1.0) \times 10^{52} \mathrm{erg}$.

\subsection{Ground-based observatories}

Following identification of the optical counterpart by UVOT (Holland et al. 2005), the field of GRB 050730 was observed by numerous ground-based telescopes. The afterglow detections in the $R$ (Sota et al. 2005), $r^{\prime}$ (Gomboc et al. 2005), $I$ and $J$ (Cobb et al. 2005) bands were soon distributed via the GRB Circular Network (GCN). Observations in the optical band with the MIKE echelle spectrograph on Magellan II led to the GRB redshift measurement $z=3.969$ (Chen et al. 2005b) based on the detection of a strong hydrogen $\operatorname{Ly} \alpha$ absorption line and of several absorption lines from other elements. The redshift was later confirmed with observations by the ISIS spectrograph on the William Herschel Telescope (Rol et al. 2005), the IMACS imaging spectrograph on the Magellan Observatory Baade Telescope (Holman et al. 2005a), FORS1 and UVES on the Very Large Telescope (D'Elia et al. 2005).

The optical afterglow decay at later times was followed by several optical telescopes. In particular, measurements were made in the $R$ band up to $\sim 4$ days after the trigger (Holman et al. 2005a,b; Burenin et al. 2005; Klotz et al. 2005; Damerdji et al. 2005; D'Elia et al. 2005; Bhatt \& Sahu 2005; Kannappan et al. 2005).

Finally, a radio afterglow with a flux density $F_{\mathrm{r}}=145 \pm 28 \mu \mathrm{Jy}$ at $8.5 \mathrm{GHz}$ was detected about 2 days after the trigger using the Very Large Array (Cameron 2005).

\subsection{Swift UVOT}

Swift UVOT began to observe the field of GRB 050730 at 20:00:22 UT, $119 \mathrm{~s}$ after the BAT trigger. The first $100 \mathrm{~s}$ exposure in the $V$ band led to the identification of the optical afterglow at $\mathrm{RA}(\mathrm{J} 2000)=14^{\mathrm{h}} 08^{\mathrm{m}} 17^{\mathrm{s}} .09, \operatorname{Dec}(\mathrm{J} 2000)=-03^{\circ} 46^{\prime} 18^{\prime \prime} \cdot 9$ (Holland et al. 2005). 
Table 1. UVOT detections of GRB 050730 in the $V$ and $B$ filters. Column (1) gives the image mid-time in seconds since the BAT trigger, Col. (2) the net exposure time, Col. (3) the filter used, and Col. (4) the afterglow magnitude with $1 \sigma$ error.

\begin{tabular}{cccc}
\hline \hline $\begin{array}{c}T(\mathrm{mid}) \\
(\mathrm{s})\end{array}$ & $\begin{array}{c}\text { Exposure } \\
(\mathrm{s})\end{array}$ & Filter & Magnitude \\
\hline 170 & 99 & $V$ & $17.4 \pm 0.2$ \\
270 & 10 & $B$ & $18.4 \pm 0.4$ \\
299 & 9.7 & $V$ & $17.2 \pm 0.5$ \\
383 & 9.7 & $V$ & $17.3 \pm 0.5$ \\
468 & 9.7 & $V$ & $16.4 \pm 0.3$ \\
524 & 30 & $B$ & $19.0 \pm 0.4$ \\
552 & 9.7 & $V$ & $16.3 \pm 0.3$ \\
637 & 9.7 & $V$ & $17.0 \pm 0.4$ \\
721 & 9.7 & $V$ & $16.8 \pm 0.4$ \\
734 & 20 & $B$ & $19.2 \pm 0.5$ \\
10164 & 900 & $B$ & $20.2 \pm 0.3$ \\
11947 & 837 & $V$ & $18.6 \pm 0.2$ \\
23519 & 835 & $V$ & $19.5 \pm 0.3$ \\
34990 & 843 & $V$ & $19.9 \pm 0.4$ \\
\hline
\end{tabular}

We refined the preliminary photometric analysis (Blustin et al. 2005) processing all UVOT data using the standard UVOT software package (Swift software v. 2.1 included in the HEAsoft package v. 6.0.2). The flux in all filters was estimated by integrating over a $3.5^{\prime \prime}$ region. A background region for subtraction of the sky contribution to the flux was selected in a relatively empty part of the field of view. The results are listed in Table 1. No significant detection was found in the $U$ and $U V$ filters, consistent with the high redshift measured for this GRB. All magnitudes are corrected for Galactic extinction $(E(B-V)=0.049$, Schlegel et al. 1998).

\subsection{Swift $X R T$}

The XRT observations of the GRB 050730 field started at 20:00:28 UT, $125 \mathrm{~s}$ after the BAT trigger, with the instrument in Auto State. After a first exposure in Image Mode (see Hill et al. 2004 for a description of readout modes) during which no on-board centroid was determined, the instrument switched into Windowed Timing (WT) mode for the entire first Swift orbit from $T_{0}+133 \mathrm{~s}$ to $T_{0}+794 \mathrm{~s}$. Starting from the second orbit $\left(T_{0}+4001 \mathrm{~s}\right)$, the instrument was in Photon Counting (PC) mode for 26 consecutive orbits until 11:49:22 UT on 2005 August 1 $\left(T_{0}+143.4 \mathrm{ks}\right)$. The field of GRB 050730 was re-observed with the XRT from August 3 starting at 15:28:11 UT $\left(T_{0}+329.4 \mathrm{ks}\right)$ until August 5 13:57:02 UT ( $\left.T_{0}+496.7 \mathrm{ks}\right)$.

The XRT data were processed with the XRTDAS software (v. 1.7.1) included in the HEAsoft package (v. 6.0.4). Event files were calibrated and cleaned with standard filtering criteria with the XRTPIPELINE task using the latest calibration files available in the Swift CALDB distributed by HEASARC. Events in the energy range $0.3-10 \mathrm{keV}$ with grades $0-12$ (PC mode) and 0-2 (WT mode) were used in the analysis (see Burrows et al. 2005a, for a definition of XRT event grades). After screening, the total exposure time for the first XRT observation was $649 \mathrm{~s}$ (WT) and $58480 \mathrm{~s}$ (PC), while for the follow-up observation the PC exposure time was $34669 \mathrm{~s}$.

In the $0.3-10 \mathrm{keV}$ PC image of the field, a previously uncatalogued X-ray source was visible within the BAT error circle with coordinates $\mathrm{RA}(\mathrm{J} 2000)=14^{\mathrm{h}} 08^{\mathrm{m}} 17^{\mathrm{s}} .2$, $\operatorname{Dec}(\mathrm{J} 2000)=-03^{\circ} 46^{\prime} 19^{\prime \prime}$. This position, derived using data not affected by pile-up (orbits 5-26, see below), has

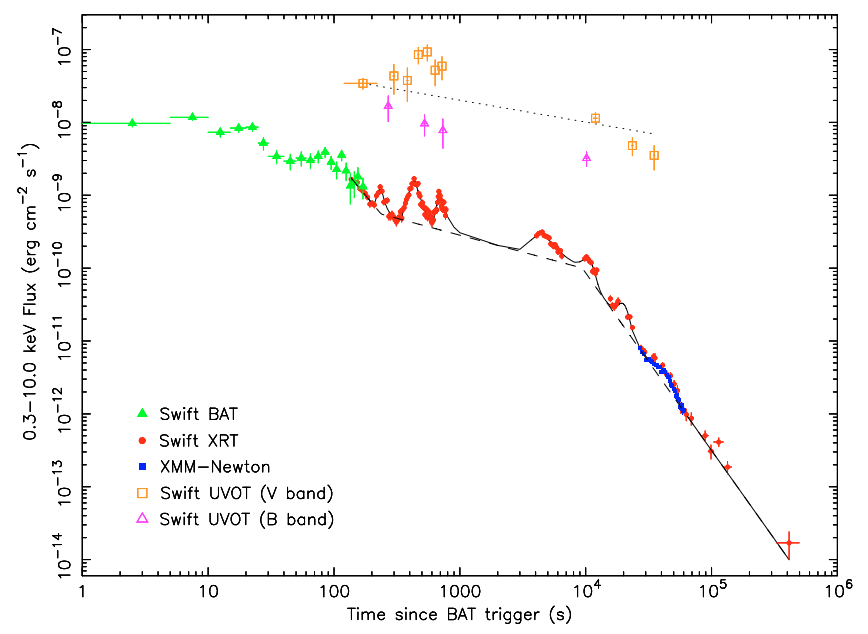

Fig. 2. Swift XRT (filled circles) and XMM-Newton (filled squares) $0.3-10 \mathrm{keV}$ light curve of the X-ray afterglow of GRB 050730. The BAT $20-150 \mathrm{keV}$ prompt emission light curve, extrapolated to the $0.3-10 \mathrm{keV}$ band, is also shown as filled triangles. UVOT optical data in the $V$ and $B$ bands, arbitrarily scaled for comparison with the X-ray band, are indicated with open squares and open triangles, respectively. The solid line is the best fit model to the XRT and XMM-Newton light curve. The dashed line represents the underlying double-broken powerlaw decay (see Sect. 3.1). The dotted line is a power-law model with temporal decay index $\alpha_{\mathrm{V}}=0.3$ normalized to fit data points in the $V$ band. Errors are at the $1 \sigma$ level.

a $90 \%$ uncertainty of $3.5^{\prime \prime}$ using the latest XRT boresight correction (Moretti et al. 2006) and is consistent with the position of the optical counterpart (Holland et al. 2005; Jacques \& Pimentel 2005).

For the WT mode data, events for temporal and spectral analysis were selected using a 40 pixel wide (1 pixel corresponds to $2.36^{\prime \prime}$ ) rectangular region centered on the afterglow and aligned along the WT one-dimensional stream in sky coordinates. Background events were extracted from a nearby sourcefree rectangular region of 50 pixel width. For PC mode data, the source count rate during orbits $2-4$ was above $\sim 0.5$ counts $\mathrm{s}^{-1}$ and data were significantly affected by pile-up in the inner part of the Point Spread Function (PSF). After comparing the observed PSF profile with the analytical model derived by Moretti et al. (2005), we removed pile-up effects by excluding events within a 5-pixel radius circle centered on the afterglow position and used an outer radius of 30 pixels. From orbit 5, the afterglow brightness was below the pile-up limit and events were extracted using a 10-pixel radius circle, which encloses about $80 \%$ of the PSF at $1.5 \mathrm{keV}$, to maximize the signal-tonoise-ratio. The background for PC mode was estimated from a nearby source-free circular region of 50-pixel radius. Source count rates for temporal analysis were corrected for the fraction of PSF falling outside the event extraction regions. Moreover, the loss of effective area due to the presence of 2 Charge Coupled Device (CCD) hot columns within the extraction regions was properly taken into account. The count rates were then converted into unabsorbed $0.3-10 \mathrm{keV}$ fluxes using the conversion factor derived from the spectral analysis (see Sect. 4).

For the spectral analysis, ancillary response files were generated with the XRTMKARF task applying corrections for the PSF losses and CCD defects. The latest response matrices (v. 008) available in the Swift CALDB were used and source spectra were binned to ensure a minimum of 20 counts per bin to utilize the $\chi^{2}$ minimization fitting technique. 


\subsection{XMM-Newton}

XMM-Newton follow-up observations of GRB 050730 started $26.4 \mathrm{ks}$ (for the two EPIC-MOS cameras) and $29.4 \mathrm{ks}$ (for the EPIC-PN) after the initial BAT Trigger. The XMM-Newton ODF (Observation Data Files) data were processed with the EPPROC and EMPROC pipeline scripts, using the XMM-Newton SAS analysis package (v. 6.5). A bright rapidly decaying source was detected near the aim-point of all three EPIC detectors and the afterglow was localized at $\mathrm{RA}(\mathrm{J} 2000)=14^{\mathrm{h}} 08^{\mathrm{m}} 17^{\mathrm{s}} .3$, $\operatorname{Dec}(\mathbf{J} 2000)=-03^{\circ} 46^{\prime} 18^{\prime \prime}$. 5 . The duration of the XMM-Newton follow-up observation was $33.7 \mathrm{ks}$ (MOS) and $30.4 \mathrm{ks}(\mathrm{PN})$. After screening out times with high background flaring, the dead-time corrected net exposures were $25.0 \mathrm{ks}$ (MOS) and $17.9 \mathrm{ks}(\mathrm{PN})$. All three EPIC cameras (PN and $2 \mathrm{MOS})$ were used in Full Window Mode, with PN and MOS2 using the "Thin" filter and MOS1 using the "Medium" optical blocking filter.

Source spectra and light curves for all 3 EPIC cameras were extracted from circular regions of $30^{\prime \prime}$ radius centered on the afterglow. Background data were taken from a $60^{\prime \prime}$ radius circle on the same chip as the afterglow, but free of any background X-ray sources. The data were further screened by including only good $\mathrm{X}$-ray events (using the selection expression FLAG $=0$ in evselect), by including events with single and double pixel events (PATTERN $<=4$ ) for the PN and by selecting single to quadruple pixel events (PATTERN $<=12$ ) for the MOS. Data below $300 \mathrm{eV}$ and above $10 \mathrm{keV}$ were also removed.

For the temporal analysis, we adopted the light curve from the MOS data, primarily because the PN data were heavily affected by background flares towards the end of the observation, while the MOS covered a wider duration at the beginning of the observation. The data from the two MOS detectors were combined and the count rate to $0.3-10 \mathrm{keV}$ unabsorbed flux conversion factor was calculated from the best fit absorbed power-law spectrum (see Sect. 4).

For the spectral analysis, ancillary and redistribution response files for fitting were generated with the SAS tasks ARFGEN and RMFGEN, respectively. Moreover, source spectra were binned to a minimum of 25 counts per bin.

\section{Temporal analysis}

The background subtracted $0.3-10 \mathrm{keV}$ Swift XRT and XMMNewton light curves of the X-ray afterglow of GRB 050730 are shown in Fig. 2. The same figure also shows the BAT 20-150 keV prompt emission light curve, converted in the $0.3-10 \mathrm{keV}$ energy band using the BAT spectral best fit model which is valid also in the XRT bandpass (see Sect. 4.1). The UVOT optical light curves in the $V$ and $B$ bands, in arbitrary units, are also plotted (see Sect. 2.3).

\subsection{X-ray afterglow}

The X-ray afterglow of GRB 050730 is characterized by a very complex structure. The first Swift orbit (from $T_{0}+133 \mathrm{~s}$ to $T_{0}+794 \mathrm{~s}$ ), after an initial steep decay phase that links well with the end of the BAT prompt emission, is dominated by three bright X-ray flares peaking at about 235, 435 and $685 \mathrm{~s}$ after the BAT trigger. Taking into account cosmological time dilation these times correspond to about 47, 88 and $138 \mathrm{~s}$ in the GRB rest frame. A flaring episode is also observed in the second orbit peaking at about $T_{0}+4500 \mathrm{~s}$. While the underlying decay of the afterglow during the first two orbits is shallow, starting from the

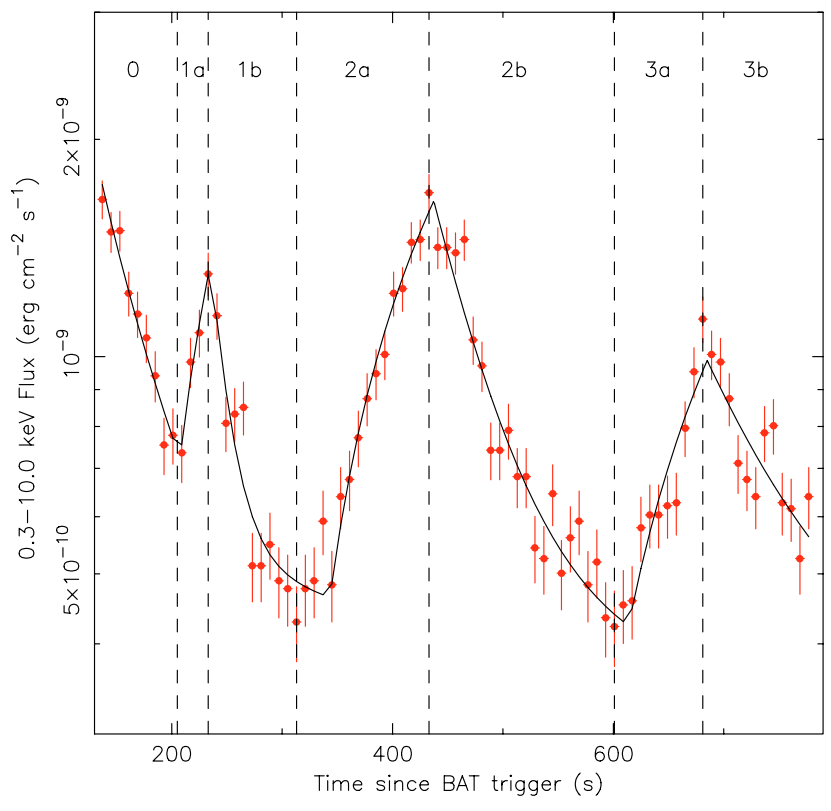

Fig. 3. Swift XRT 0.3-10 keV light curve of the GRB 050730 X-ray afterglow during the first orbit. The solid line is the best fit model for the data obtained considering a linear rise exponential decay for the three flares (see Sect. 3.1). The dashed vertical lines delimit the seven time-intervals considered for the spectral analysis. Data are binned to $8 \mathrm{~s}$ resolution and errors are at the $1 \sigma$ level.

third orbit $\left(T_{0}+10 \mathrm{ks}\right)$ the afterglow light curve shows a much steeper decline with superimposed flaring activity.

We first modeled the X-ray light curve of the afterglow with a double broken power-law model with slopes $\alpha_{1}, \alpha_{2}, \alpha_{3}$ and temporal breaks $t_{1}, t_{2}$, describing the underlying power-law decay of the afterglow, plus seven Gaussian functions modeling the flaring episodes. We found for the first power-law a decay index $\alpha_{1}=2.1 \pm 0.3$ followed, after a first time break at $t_{1}=237 \pm 20 \mathrm{~s}$, by a shallower decay with index $\alpha_{2}=0.44_{-0.08}^{+0.14}$. A second temporal break is found at $t_{2}=10.1_{-2.2}^{+4.6} \mathrm{ks}$ after which a steep decay with index $\alpha_{3}=2.40_{-0.07}^{+0.09}$ is observed. This model did not provide a good fit $\left(\chi_{r}^{2}=1.73,143\right.$ d.o.f.), mostly due to short time scale fluctuations and to deviations of the three first bright flares from a symmetric Gaussian shape.

We thus considered a different functional form for the three X-ray flares, namely a linear rise exponential decay: $F(t) \propto$ $\left(t-t_{0}\right) /\left(t_{\mathrm{p}}-t_{0}\right)$ for times between the flare start time $t_{0}$ and peak time $t_{\mathrm{p}}, F(t) \propto \exp \left[-\left(t-t_{\mathrm{p}}\right) / t_{\mathrm{c}}\right]$ for $t>t_{\mathrm{p}}$ where $t_{\mathrm{c}}$ is the exponential decay time. The model improves significantly the fit with $\chi_{r}^{2}=1.43$ (140 d.o.f.) and F-test chance probability of $1.3 \times 10^{-6}$. The best fit parameters of the overall underlying double broken power-law model were unchanged with respect to the previous fit listed above. The linear rise exponential decay best fit parameters for the first three flares are given in Table 2 while Table 3 reports the Gaussian best fits for the other four flares. As Fig. 3 illustrates, the second brightest flare (referred to as flare 2 in the following) has a flux variation of amplitude $~ 3.6$ and is characterized by a steep rise, lasting $\sim 90 \mathrm{~s}$, followed by a slower decay with duration $\sim 170 \mathrm{~s}$. An asymmetric shape, with a steep rise followed by a shallower decay, was also observed for the other two flares (flares 1 and 3 ) and for these episodes the flux variation was $\sim 1.7$ and $\sim 2.6$, respectively.

The ratio between the duration $(\Delta t)$ and the peak time $\left(t_{\mathrm{p}}\right)$ of the $X$-ray flares was calculated using the linear rise exponential decay and Gaussian best fit parameters for the first three and 


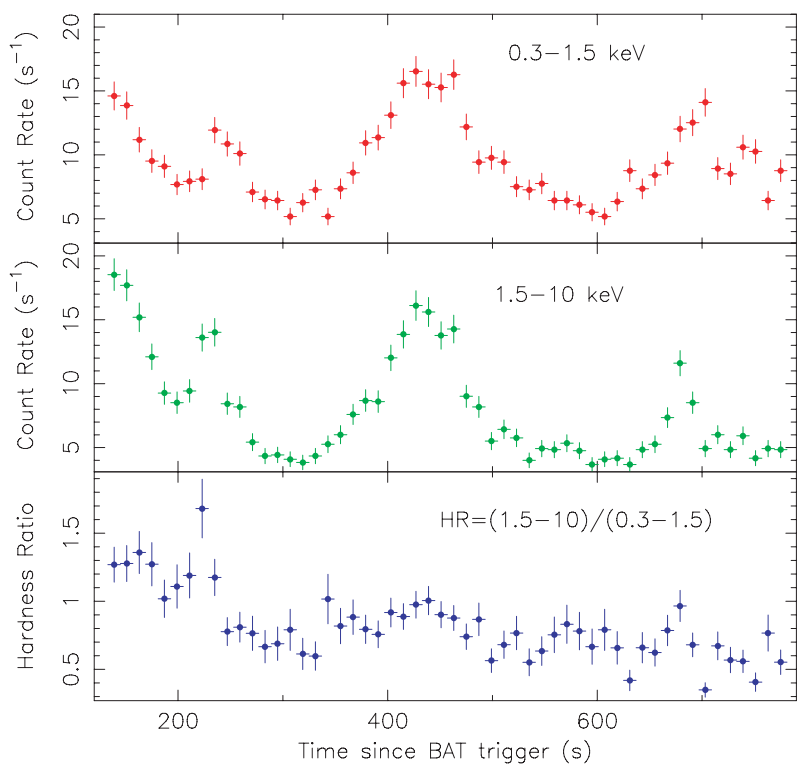

Fig. 4. Swift XRT 0.3-1.5 keV (upper panel) and 1.5-10 keV (middle panel) light curve of the GRB $050730 \mathrm{X}$-ray afterglow during the first Swift orbit. In the lower panel the corresponding hardness ratio is plotted. Data are binned to $12 \mathrm{~s}$ resolution and error bars indicate statistical uncertainties at the $1 \sigma$ level.

Table 2. Temporal best fit parameters of the first three bright X-ray flares of GRB 050730 using a linear rise exponential decay model. The corresponding $\Delta t / t_{\mathrm{p}}$ values for the three flares are also shown (see Sect. 3.1).

\begin{tabular}{cccccc}
\hline \hline Flare & $t_{0}$ & $t_{\mathrm{p}}$ & $t_{\mathrm{c}}$ & $K \times 10^{-10}$ & $\Delta t / t_{\mathrm{p}}$ \\
& $(\mathrm{s})$ & $(\mathrm{s})$ & $(\mathrm{s})$ & $\left(\mathrm{erg} \mathrm{cm}^{-2} \mathrm{~s}^{-1}\right)$ & \\
\hline 1 & $207_{-5}^{+5}$ & $236_{-4}^{+3}$ & $17_{-5}^{+6}$ & $8 \pm 1$ & $0.3 \pm 0.1$ \\
2 & $344_{-5}^{+5}$ & $437_{-4}^{+5}$ & $54_{-6}^{+6}$ & $12.2 \pm 0.7$ & $0.58 \pm 0.04$ \\
3 & $614_{-10}^{+9}$ & $685_{-6}^{+7}$ & $87_{-21}^{+37}$ & $6.1 \pm 0.7$ & $0.5 \pm 0.1$ \\
\hline
\end{tabular}

Table 3. Temporal best-fit parameters of the X-ray flares 4-6 and 7 of GRB 050730 using Gaussian functions. An asterisk indicates a frozen parameter. The corresponding $\Delta t / t_{\mathrm{p}}$ values are also indicated (see Sect. 3.1). Best-fit parameters for flare 7 were derived from $X M M$ Newton data only.

\begin{tabular}{ccccc}
\hline \hline Flare & $\begin{array}{c}t_{\mathrm{p}} \\
(\mathrm{s})\end{array}$ & $\begin{array}{c}\sigma \\
(\mathrm{s})\end{array}$ & $\begin{array}{c}K \times 10^{-11} \\
\left(\mathrm{erg} \mathrm{cm}^{-2} \mathrm{~s}^{-1}\right)\end{array}$ & $\Delta t / t_{\mathrm{p}}$ \\
\hline 4 & $4484_{-235}^{+124}$ & $641_{-149}^{+259}$ & $11 \pm 2$ & $0.7 \pm 0.2$ \\
5 & $10391_{-315}^{+330}$ & $1500^{*}$ & $5.5 \pm 1.0$ & $0.71 \pm 0.02$ \\
6 & $18714_{-1156}^{+952}$ & $3638_{-789}^{+820}$ & $1.5 \pm 0.4$ & $0.9 \pm 0.2$ \\
7 & $41244_{-744}^{+691}$ & $6170_{-718}^{+819}$ & $0.12 \pm 0.02$ & $0.7 \pm 0.1$ \\
\hline
\end{tabular}

the last four flares, respectively. For both analytical models the duration of a flare was defined as the time interval during which the flare intensity was above $5 \%$ of its peak value. The results are given in Tables 2 and 3.

The rise and decay portions of the first three flares were also fit with single power-laws. For the estimation of power-law indices, times were expressed from the onset of the flares $\left(t_{0}\right)$ for the rise and from the peak time $\left(t_{\mathrm{p}}\right)$ for the decay. The contribution of the underlying afterglow decay was taken into account.
Table 4. Best fit temporal indices of the rising $\left(\alpha_{\mathrm{r}}\right)$ and decaying $\left(\alpha_{\mathrm{d}}\right)$ portions of the X-ray flares 1, 2, 3 and 7 using a single power-law model.

\begin{tabular}{lcc}
\hline \hline Flare & $\alpha_{\mathrm{r}}$ & $\alpha_{\mathrm{d}}$ \\
\hline 1 & $-1.56 \pm 0.69$ & $1.25 \pm 0.32$ \\
2 & $-1.84 \pm 0.30$ & $1.31 \pm 0.44$ \\
3 & $-1.46 \pm 0.62$ & $0.75 \pm 0.35$ \\
7 & $-0.89 \pm 0.34$ & $1.61 \pm 0.44$ \\
\hline
\end{tabular}

The power-law temporal indices for the rising $\left(\alpha_{\mathrm{r}}\right)$ and decaying $\left(\alpha_{\mathrm{d}}\right)$ portions of the three flares are reported in Table 4.

The temporal behavior of the afterglow during the first three $\mathrm{X}$-ray flares was also studied in different energy bands. In Fig. 4 we show the $0.3-1.5 \mathrm{keV}$ (upper panel) and 1.5-10 keV (middle panel) light curves of the early X-ray afterglow together with the corresponding hardness ratio (lower panel). From the figure it is apparent that i) in the harder band the profile of the flares is sharper and ii) a peak time shift, with flares in the hard band peaking at earlier times, is observed. We note that these temporal properties as a function of energy are the same observed for the prompt emission pulses of GRBs.

As already mentioned, late flaring activity of the X-ray afterglow was observed at $\sim 4.5,10.4,18.7$ and $41.2 \mathrm{ks}$ after the trigger. Due to the Swift orbital gaps the temporal coverage of flares 4, 5 and 6 is poor and these episodes could not be properly studied, but the last flare was entirely covered by the $X M M$ Newton follow-up observation allowing a detailed temporal analysis. In Fig. 5 the XMM-Newton (MOS1+MOS2) light curve is shown together with the XRT curve covering the same time interval. Good agreement between the two curves is found and the $\sim 5 \%$ higher normalization of the XRT data points is of the same order of the uncertainties in the absolute flux calibration of the instrument (Campana et al. 2006).

We first fit the XMM-Newton light curve with a single powerlaw decay and obtained an extremely poor fit to the light curve, with a fit statistic of $\chi_{r}^{2}=8.81$ (32 d.o.f.) and a decay index of $\alpha=2.10 \pm 0.04$. The light curve was then parameterized with a long duration flare super-imposed on a steep power-law decay. We obtained an underlying decay index $\alpha=2.45 \pm 0.15$, while the flare could be adequately modeled with a Gaussian function; the fit statistic was acceptable $\left(\chi_{r}^{2}=1.22,29\right.$ d.o.f.). The long duration flare peaked at $41 \mathrm{ks}$ (or $8.2 \mathrm{ks}$ in the GRB rest frame), with a $\sigma$ of $6.8 \mathrm{ks}(1.4 \mathrm{ks}$ rest frame). The total fluence of the flare when parameterized this way was $8.3 \times 10^{50} \mathrm{erg}(1.5-50 \mathrm{keV}$ band in the GRB rest frame), which represents $20 \%$ of the integrated afterglow emission over the XMM-Newton observation. The rise and decay phases of flare 7 were also fit with single power-laws expressing times from the onset and from the peak time of the flare, respectively. The measured temporal indices $\alpha_{\mathrm{r}}$ and $\alpha_{\mathrm{d}}$ are also reported in Table 4.

\subsection{Optical band}

The UVOT optical light curve of the afterglow of GRB 050730 in the $V$ and $B$ bands is also characterized by a complex behavior, as illustrated in Fig. 2.

The early $\left(T_{0}+100 \mathrm{~s}-T_{0}+800 \mathrm{~s}\right)$ UVOT $V$ light curve reveals flaring activity. A re-brightening is observed at $\sim T_{0}+500 \mathrm{~s}$, almost simultaneously with the brightest X-ray flare observed with XRT. Due to the relatively poor sampling of the UVOT light curve, a detailed temporal analysis is not possible, however we note that the amplitude of the flux variation in the $V$ band $(\sim 3)$ is of the same order as that measured in the X-ray energy 


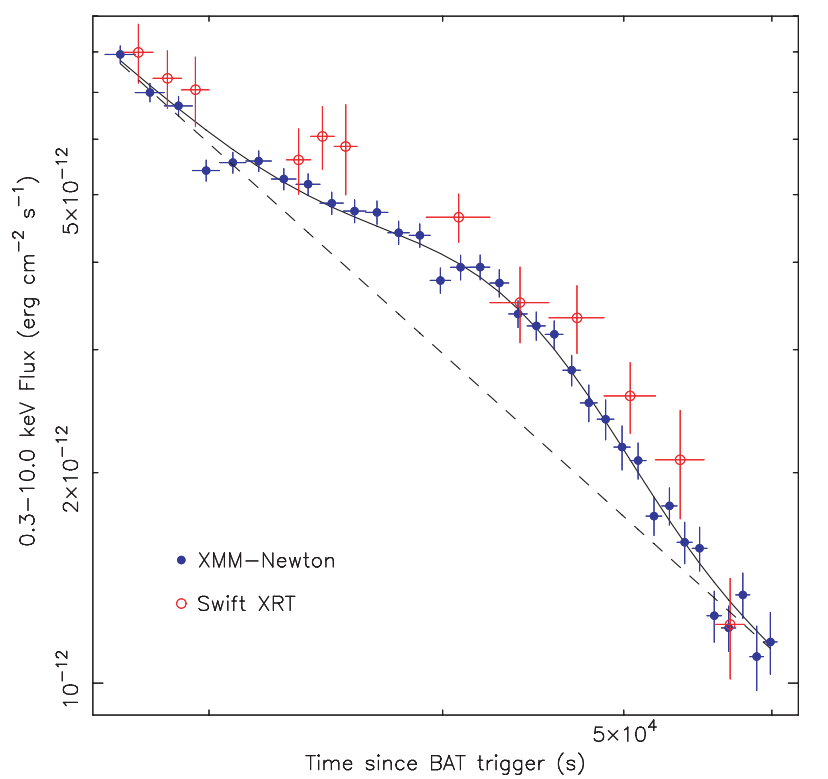

Fig. 5. XMM-Newton (MOS1+MOS2, filled circles) and Swift XRT (open circles) $0.3-10 \mathrm{keV}$ light curve of the late $\left(\sim T_{0}+12 \mathrm{~h}\right) \mathrm{X}$-ray afterglow of GRB 050730. The solid line is the best fit model to the $X M M$-Newton light curve. The dashed line represents the underlying power-law decay (see Sect. 3.1). Data points errors are at the $1 \sigma$ level.

band. The decay of the afterglow in the $V$ band, up to about $T_{0}+12 \mathrm{ks}$ and excluding the re-brightening episode, is shallow $\left(\alpha_{\mathrm{V}} \sim 0.3\right)$ and in agreement with that measured in the X-ray band $\left(\alpha_{\mathrm{X}}=0.44_{-0.08}^{+0.14}\right)$. Hints of a steepening of the $V$ light curve at $\sim T_{0}+12 \mathrm{ks}$ are also found, but the limited temporal coverage and the large statistical uncertainties characterizing the UVOT data points do not allow us to constrain the late optical afterglow decay.

The UVOT light curve in the $B$ band is characterized by a flat power-law decay ( $\left.\alpha_{\mathrm{B}} \sim 0.3\right)$ without re-brightening events. However, it should be noted that i) the curve is very sparsely sampled, with only three data points during the first Swift orbit, and ii) in the $B$ band there is a strong flux reduction due to the Lyman break and thus large statistical uncertainties affect the data points.

Accurate optical observations of the afterglow of GRB 050730 in the $R$ and $I$ bands are presented by Pandey et al. (2006). The authors report an early time flux decay with indices $\alpha_{\mathrm{R}}=0.54 \pm 0.05$ and $\alpha_{\mathrm{I}}=0.66 \pm 0.11$ followed, after a temporal break at about $9 \mathrm{ks}$ after the trigger, by a strong steepening with decay indices $\alpha_{\mathrm{R}}=1.75 \pm 0.05$ and $\alpha_{\mathrm{I}}=1.66 \pm 0.07$.

\section{Spectral analysis}

For the spectral analysis of Swift XRT and XMM-Newton data, we used the XSPEC package (v. 11.3.2, Arnaud 1996) included in the HEAsoft package (v. 6.0.4).

\section{1. $X R T$}

As a first step, the $0.3-10 \mathrm{keV}$ XRT average spectrum during the first Swift orbit (WT mode, from $T_{0}+133 \mathrm{~s}$ to $T_{0}+794 \mathrm{~s}$ ) was fit adopting a single power-law model with the neutral hydrogen-equivalent absorption column density fixed at the Galactic value in the direction of the GRB $\left(N_{\mathrm{H}}^{\mathrm{G}}=3.0 \times\right.$ $10^{20} \mathrm{~cm}^{-2}$, Dickey \& Lockman 1990). The fit obtained was poor
Table 5. Results of single power-law spectral fits to the $0.3-10 \mathrm{keV}$ spectrum of the afterglow of GRB 050730. A local $(z=0)$ absorption column fixed at the known Galactic value of $N_{\mathrm{H}}^{\mathrm{G}}=3.0 \times 10^{20} \mathrm{~cm}^{-2}$ (Dickey \& Lockman 1990) was used in the fits. An asterisk indicates a frozen parameter.

\begin{tabular}{|c|c|c|c|c|}
\hline Segment & $\begin{array}{c}\text { Time interval } \\
\text { (s) }\end{array}$ & $\begin{array}{l}N_{\mathrm{H}}^{z} \times 10^{22} \\
\left(\mathrm{~cm}^{-2}\right)\end{array}$ & $\beta$ & $\chi_{r}^{2}$ (d.o.f.) \\
\hline WT (all) & $133-781$ & $1.28_{-0.25}^{+0.26}$ & $0.70_{-0.03}^{+0.03}$ & $1.01(310)$ \\
\hline WT (0) & $133-205$ & $1.8_{-0.8}^{+0.9}$ & $0.42_{-0.08}^{+0.08}$ & $0.86(76)$ \\
\hline WT (1a) & $205-233$ & $1.6_{-1.6}^{+2.5}$ & $0.29_{-0.16}^{+0.16}$ & $0.86(24)$ \\
\hline WT (1b) & $233-313$ & $3.1_{-1.1}^{+1.3}$ & $0.82_{-0.12}^{+0.12}$ & $1.30(51)$ \\
\hline WT (2a) & $313-433$ & $2.1_{-0.7}^{+0.8}$ & $0.71_{-0.08}^{+0.08}$ & $1.10(93)$ \\
\hline WT (2b) & $433-601$ & $0.9_{-0.5}^{+0.5}$ & $0.70_{-0.07}^{+0.07}$ & $1.11(111)$ \\
\hline WT (3a) & $601-681$ & $0.7_{-0.7}^{+0.8}$ & $0.77_{-0.12}^{+0.12}$ & $0.87(46)$ \\
\hline WT (3b) & $681-781$ & $1.0_{-0.6}^{+0.6}$ & $1.01_{-0.10}^{+0.10}$ & $0.81(65)$ \\
\hline PC (1) & $4001-18149$ & $1.1_{-0.4}^{+0.4}$ & $0.61_{-0.04}^{+0.04}$ & $0.95(224)$ \\
\hline $\mathrm{PC}(2)$ & $21288-143438$ & $1.0_{-0.6}^{+0.7}$ & $0.81_{-0.08}^{+0.08}$ & $1.16(86)$ \\
\hline XMM (all) & $29436-59811$ & $0.68_{-0.10}^{+0.10}$ & $0.87_{-0.02}^{+0.02}$ & $1.14(489)$ \\
\hline XMM (1) & $29436-40000$ & $0.68^{*}$ & $0.87_{-0.03}^{+0.03}$ & $0.88(345)$ \\
\hline XMM (2) & $40000-50000$ & $0.68^{*}$ & $0.93_{-0.03}^{+0.03}$ & $0.98(243)$ \\
\hline XMM (3) & $50000-59811$ & $0.68^{*}$ & $0.99_{-0.05}^{+0.05}$ & $0.77(135)$ \\
\hline
\end{tabular}

$\left(\chi_{\mathrm{r}}^{2}=1.28,311\right.$ d.o.f.). From the inspection of residuals a strong deficit of counts at low energies, likely due to the presence of an absorbing column density in excess of that of the Galactic one, was found. Indeed, the addition of a column density $N_{\mathrm{H}}^{z}$ using Solar metallicity redshifted to the rest frame of the GRB host $(z=3.969)$ free to vary in the spectral fit (ZWABS model in XSPEC) resulted in a more acceptable fit with a column density of $N_{\mathrm{H}}^{z}=\left(1.28_{-0.25}^{+0.26}\right) \times 10^{22} \mathrm{~cm}^{-2}$ (see Table 5). This value is consistent with the neutral hydrogen column densities derived from the optical spectra reported by Starling et al. (2005) and Chen et al. (2005a).

From Fig. 4 it is apparent that strong spectral evolution takes place during the intense flaring activity observed in the first Swift orbit. We thus split the WT observation into seven time intervals to study the spectra during the rise and decay portions of each flare (see Fig. 3). The time-resolved spectral best fits (see Table 5) clearly show evidence for spectral variation during the flares and an overall softening of the spectra with time associated with a decrease of the rest frame column density. The timeresolved spectral analysis was also performed adopting a broken power-law model to investigate the possible presence of spectral breaks (e.g. Falcone et al. 2006; Guetta et al. 2007). Also in this case an additional absorption column density $N_{\mathrm{H}}^{z}$ at the rest frame of the GRB host was considered. We did not find evidence for spectral breaks within the XRT energy band for any segments.

The XRT late $0.3-10 \mathrm{keV}$ spectrum (PC mode, from $T_{0}+4.0 \mathrm{ks}$ to $T_{0}+143.4 \mathrm{ks}$ ) was also fit using a single powerlaw model with a fixed Galactic absorption column density and an additional absorbing column at the burst rest frame. The observation was divided in two time intervals (4.0-18.1 ks and 21.3-143.4 ks from the trigger). The results are listed in Table 5 
where spectral softening between the two time intervals was identified.

\subsection{XMM-Newton}

The PN and MOS 0.3-10 keV spectra were fit jointly allowing the cross normalization between the detectors, which is consistent within $<5 \%$, free to vary. The two MOS spectra and responses were combined to maximize the signal to noise, after first checking that they were consistent with each other.

As in the case of the XRT spectrum, the PN and MOS spectra were first fit with a single power-law model with the neutral hydrogen-equivalent absorption column fixed at the known Galactic value. The fit obtained was not acceptable $\left(\chi_{\mathrm{r}}^{2}=1.40\right.$, 489 d.o.f.), while the energy index was $\beta=0.76 \pm 0.02$. The addition of a neutral absorption column in the GRB host galaxy frame at $z=3.969$ resulted in a more acceptable fit $\left(\chi_{\mathrm{r}}^{2}=1.14\right.$, 489 d.o.f.) with an excess absorption column above the Galactic column of $(6.8 \pm 1.0) \times 10^{21} \mathrm{~cm}^{-2}$, while the continuum energy index was $\beta=0.87 \pm 0.02$.

The XMM-Newton afterglow spectra were also sliced into three segments of approximately $10 \mathrm{ks}$ in duration, to search for any spectral evolution within the XMM-Newton observation. A small change in the continuum parameters was found, the spectrum evolved from hard to soft; the energy index changed from $\beta=0.87 \pm 0.03$ in the first $10 \mathrm{ks}$, to $\beta=0.99 \pm 0.05$ during the final segment. No evidence was found for a change in the column density, which was subsequently fixed at $N_{\mathrm{H}}=6.8 \times 10^{21} \mathrm{~cm}^{-2}$ in all the segments. The spectral best fit parameters are shown in Table 5. We also searched for any evidence of emission lines in the mean spectrum and in the three segments. No statistically significant lines were found, at the level of $>99 \%$ confidence. As the redshift of the burst is known, then we can set an upper-limit to the equivalent width of any emission lines. Over the range of $0.4-8 \mathrm{keV}$ and using the mean spectrum, we found a $<100 \mathrm{eV}$ upper limit to all emission lines. More specifically we can set a limit on the iron $\mathrm{K} \alpha$ line (e.g. the $\mathrm{H}$-like line at $6.97 \mathrm{keV}$ rest frame, $1.40 \mathrm{keV}$ observed frame) of $<30 \mathrm{eV}$.

\section{Discussion}

\subsection{Early $X$-ray light curve}

This GRB, with its rather high redshift, $z=3.969$, gives us the possibility to investigate the X-ray and optical light curves soon after the trigger and thus to study in detail the soft tail of the prompt emission and the very beginning of the afterglow phase. Indeed, due to cosmological time dilation, the XRT and UVOT observations started, in the rest frame of the burst, only $27 \mathrm{~s}$ after the trigger. Other examples of early observations of high redshift bursts are GRB $050904(z=6.29$, Cusumano et al. 2006b) and GRB 060206 ( $z=4.0$, Monfardini et al. 2006).

The XRT light curve shows at the very beginning (133-205 s from the BAT trigger) a rapidly decaying emission that links well with the BAT flux when converted to the XRT bandpass, a feature that has been observed in various Swift bursts (Tagliaferri et al. 2005; Barthelmy et al. 2005b; Nousek et al. 2006; O'Brien et al. 2006). For GRB 050730, the steep temporal decay index $\left(\alpha_{1}=2.1 \pm 0.3\right.$, see Sect. 3.1) characterizing the early X-ray light curve suggests that the observed emission is probably associated with the tail of the prompt emission rather than with the beginning of the shocked inter-stellar medium afterglow phase. Indeed, in the internal shock model scenario for the prompt $\gamma$-ray emission (e.g.
Rees \& Mészáros 1994), the cessation of the emissivity is characterized by a rapid decay due to the delayed arrival of the high angular latitude prompt emission of the shocked surface (high-latitude or curvature emission, Kumar \& Panaitescu 2000; Dermer 2004). The high-latitude emission predicts a relationship between the temporal decay index $\alpha_{\mathrm{d}}$ and the spectral in$\operatorname{dex} \beta_{\mathrm{d}}$ during the decay given by the equation $\alpha_{\mathrm{d}}=2+\beta_{\mathrm{d}}$ (Kumar \& Panaitescu 2000) where the decay slope is measured using as zero time $\left(t_{0}\right)$ the beginning of the prompt emission $\left(F(t, v) \propto\left(t-t_{0}\right)^{-\alpha_{\mathrm{d}}} v^{-\beta_{\mathrm{d}}}\right)$. We have tested this prediction by fitting the early XRT light curve (segment "O" in Fig. 3) with the above constraint using the spectral index measured during the decay $\left(\beta_{\mathrm{d}}=0.42 \pm 0.08\right.$, see Table 5$)$, and leaving the zero time as a free parameter (see Liang et al. 2006 for a description of the method). We found $t_{0}=-31_{-31}^{+24} \mathrm{~s}$, i.e. zero time is located at the rising segment of the BAT prompt emission light curve (see Fig. 1), as expected in the framework of the high-latitude emission. This result indicates that the steep decay observed in the early XRT light curve is most likely the soft tail of the BAT emission.

This hypothesis is also supported by the BAT and early (segment "0" in Fig. 3) XRT spectra. We found very similar spectral slopes $\left(\beta_{\mathrm{BAT}}=0.5 \pm 0.1\right.$ and $\beta_{\mathrm{XRT}}=0.42 \pm 0.08$, respectively), likely indicating that the early X-ray and $\gamma$-ray emissions are produced by the same emission mechanism.

\subsection{Flaring activity}

The exceptionally extended flaring activity of the X-ray afterglow of GRB 050730 allowed us to study this phenomenon over more than two orders of magnitude in time. The XRT early steep decay is followed by three bright X-ray flares peaking at 236, 437 and $685 \mathrm{~s}$ after the BAT trigger (see Fig. 3). These flares were under the sensitivity of the BAT instrument and thus were not detected in the hard X-ray energy band. These flares show, as other strong flares observed with Swift (e.g. Romano et al. 2006; Falcone et al. 2006; Pagani et al. 2006; Godet et al. 2006; Chincarini et al. 2006), a clear spectral evolution with the hardness ratio that mimics the variation of the light curve. A phase lag, with the harder $(E>1.5 \mathrm{keV})$ light curve peaking at earlier times with respect to the softer energy band is also found (see Fig. 4).

So far, most of the X-ray flares observed in Swift X-ray light curves have been interpreted as being due to late internal shocks (e.g. Burrows et al. 2005b; Romano et al. 2006; Falcone et al. 2006; Godet et al. 2006). In this scenario, the GRB central engine is active far beyond the end of the prompt $\gamma$ ray emission phase requiring new mechanisms capable of powering new relativistic outflows at late-time (e.g. King et al. 2005; Perna et al. 2006). A diagnostic to check if the re-brightenings are due to late internal shocks has been recently proposed by Liang et al. (2006). In the internal-origin scenario for X-ray flares the decay emission of the flaring episodes should be dominated by high-latitude emission with the decay temporal index related to the decay spectral index as $\alpha_{\mathrm{d}}=\beta_{\mathrm{d}}+2$ (Kumar \& Panaitescu 2000). We have checked this hypothesis for the first three bright X-ray flares observed in the GRB 050730 afterglow. The spectral indices measured during the decays were used (segments " $1 b$ ", " $2 b$ " and " $3 b$ ", see Fig. 3 and Table 5) and we found best fit zero times $t_{0,1}=193_{-11}^{+8} \mathrm{~s}, t_{0,2}=341_{-42}^{+29} \mathrm{~s}$ and $t_{0,3}=592_{-93}^{+35} \mathrm{~s}$, respectively. We can see that for all three episodes the zero time values are located at the beginning of the corresponding flare and thus the observed decay slopes are 
consistent with being due to high-latitude emission as predicted by the late internal shock scenario.

Another possibility is that the X-ray flares are produced by refreshed shocks (Rees \& Mészáros 1998; Kumar \& Piran 2000; Sari \& Mészáros 2000). In the standard internal-external fireball model (e.g. Rees \& Mészáros 1994; Sari \& Piran 1997) re-brightening episodes in the afterglow light curve are explained as slow shells ejected from the central engine during the prompt phase that catch up with the main afterglow shock after it has decelerated in the external inter-stellar medium. Indeed, Guetta et al. (2007) have recently interpreted the Xray flares of the afterglow of GRB 050713A as being due to refreshed shocks. Here we applied the diagnostic proposed for GRB 050713A to the case of GRB 050730. First, to estimate the rise and decay slopes of the early flares we operationally selected as zero time the beginning and the peak of the flare, respectively. This assumption implicitly means that the flare is completely independent of the main event generating the prompt emission and the forward shock light curve. We found temporal indices in the 0.8-1.8 range (see Table 4). Much steeper slopes $(\sim 3-6)$ are obtained if times are referenced from the BAT trigger, as is usually done for most Swift X-ray flares (e.g. Burrows et al. 2005b; Romano et al. 2006; Falcone et al. 2006). Second, we checked if the temporal and the spectral indices during the decay phase are related as predicted by the standard afterglow model (Sari et al. 1998). We restricted the analysis to the episodes with enough statistics to allow a detailed temporal and spectral study, i.e. the first three flares and the last one. For the decay phases we expect the following relations to hold (Sari et al. 1998; Dai \& Cheng 2001): $F_{v} \propto$ $\left(v_{\mathrm{m}} / v_{\mathrm{c}}\right)^{-1 / 2}\left(v / v_{\mathrm{m}}\right)^{-p / 2} F_{v, \text { max }} \propto t^{-3 p / 4+1 / 2}$ (for $\left.p>2\right)$ and $F_{v} \propto$ $\left(v_{\mathrm{m}} / v_{\mathrm{c}}\right)^{(p-1) / 2}\left(v / v_{\mathrm{c}}\right)^{-p / 2} F_{v, \max } \propto t^{-(3 p+10) / 16}$ (for $\left.1<p<2\right)$. From the best fit spectral energy indices during the decay of the four flares (see Table 5) we derived the predicted temporal slopes $\alpha_{\mathrm{d}}^{1}=0.93 \pm 0.05, \alpha_{\mathrm{d}}^{2}=0.89 \pm 0.03, \alpha_{\mathrm{d}}^{3}=1.02 \pm 0.15$, and $\alpha_{\mathrm{d}}^{7}=1.40 \pm 0.05$, which are consistent with the measured values of the decay indices listed in Table 4 . We note that for the last flare, peaking at $41.2 \mathrm{ks}$ after the trigger, we used the afterglow model relations for the slow cooling case $\left(F_{v} \propto\left(v / v_{\mathrm{m}}\right)^{-(p-1) / 2} F_{v, \max } \propto t^{3(1-p) / 4}\right)$ which likely is to apply at these late times (Sari et al. 1998).

Important information on the mechanism producing the flares can also be obtained from the comparison between the observed variability timescale and the time at which the flare is observed (Ioka et al. 2005). We calculated, for all 7 re-brightenings of the GRB 050730 afterglow, the ratio between the duration of the flare $(\Delta t)$ and the time when the flare peaks $\left(t_{\mathrm{p}}\right)$ (see Tables 2 and 3). We found $\Delta t / t_{\mathrm{p}} \sim 0.3$ for the first flare and $\Delta t / t_{\mathrm{p}} \sim 0.6-$ 0.7 for the others, in agreement with the $\Delta t / t_{\mathrm{p}}>0.25$ limit discussed by Ioka et al. (2005) for the refreshed shock scenario. Remarkably, the $\Delta t / t_{\mathrm{p}}$ ratio is nearly constant for all flares, with the exception of the first one which occurred during the bright and steep tail of the prompt emission (see Fig. 3) and most likely we are observing only the tip of the flare. Duration of the flare proportional to $t_{\mathrm{p}}$, as observed in the afterglow of GRB 050730, is explained both in the refreshed shock scenario (Kumar \& Piran 2000) and in the late internal shock model (Perna et al. 2006). We note that the last four flares have $\Delta t / t_{\mathrm{p}}$ values in the range $0.7-0.9$, while for flares 2 and 3 we observe slightly lower ratios $(0.5-0.6)$, possibly indicating a moderate temporal evolution of $\Delta t / t_{\mathrm{p}}$. However, due to the relatively large error bars, the measured values are also consistent with being constant and $\sim 0.6-0.7$.
Flaring activity is also observed in the optical afterglow of GRB 050730. Indeed, the UVOT $V$ early light curve (see Fig. 2) shows a re-brightening at $\sim T_{0}+500 \mathrm{~s}$, almost simultaneously with the brightest X-ray flare observed with the XRT peaking at $T_{0}+437 \mathrm{~s}$. Moreover, the optical re-brightening amplitude $(\sim 3)$ is of the same order of the flux variation observed in the X-ray energy band. Strong indications of correlated variability between the X-ray and optical energy bands are also present in the NIR/Optical light curves reported by Pandey et al. (2006): a significant re-brightening at about $T_{0}+4 \mathrm{ks}$ ( $R$ filter), close to the X-ray flare peaking at $T_{0}+4.5 \mathrm{ks}$ (see Fig. 2), is observed. Moreover, a bump in the $J, I, R$ and $V$ light curves at about $T_{0}+10 \mathrm{ks}$ is observed (Pandey et al. 2006), again simultaneous with the X-ray flare peaking at $T_{0}+10.4 \mathrm{ks}$. Although the X-ray flaring activity is not uniformly covered by optical observations, we find several indications of simultaneous re-brightening events in the X-ray and optical bands, in agreement with the refreshed shock model (e.g. Granot et al. 2003).

\subsection{Evidence of a jet break}

The X-ray afterglow light curve shows a clear temporal break around $10 \mathrm{ks}$ after the trigger (see Sect. 3.1). At about the same time a strong steepening of the $I$ and $R$ afterglow light curves, although with a shallower post-break slope, is also observed (see Pandey et al. 2006 for a detailed analysis on the discrepancy between the X-ray and optical slopes). Due to the achromatic nature of the temporal break it is very likely that a jet break is occurring when the bulk Lorentz factor $\gamma$ of the collimated relativistic outflow becomes lower than the inverse of the jet opening angle $\theta_{\text {jet }}$ (e.g., Rhoads 1997; Sari et al. 1999), as reported by Pandey et al. (2006). In this framework, the jet opening angle can be determined through the equation $\theta_{\text {jet }}=$ $0.161\left[t_{\mathrm{b}} /(1+z)\right]^{3 / 8}\left(n \eta / E_{\text {iso }}\right)^{1 / 8}$ (e.g., Bloom et al. 2003) where $\theta_{\text {jet }}$ is in radians, the jet temporal break $t_{\mathrm{b}}$ in days, the total isotropic-equivalent energy $E_{\text {iso }}$ in units $10^{52} \mathrm{erg}$, the density $n$ of the circumburst medium in $\mathrm{cm}^{-3}$ and $\eta$ is the efficiency of conversion of the outflow kinetic energy in electromagnetic radiation.

An accurate estimation of the bolometric isotropicequivalent energy radiated by GRBs requires the knowledge of their intrinsic spectrum over a broad energy band (Bloom et al. 2001; Amati et al. 2002). For GRB 050730, the spectrum is well fit by a single power-law with energy index $\beta_{\mathrm{BAT}}=0.5 \pm 0.1$ up to the high energy limit of the BAT sensitivity bandpass $(150 \mathrm{keV})$ indicating that i) we are observing the low energy tail of the Band model (Band et al. 1993) generally used to describe GRB spectra and ii) the $v F(v)$ spectrum peak energy $E_{\mathrm{p}}$ is above $\sim 750 \mathrm{keV}$ (i.e. $(1+z) \times 150 \mathrm{keV}$ ) in the burst rest frame. A lower limit to the bolometric isotropicequivalent radiated energy $E_{\text {iso }}$ is given by the observed radiated energy in the BAT bandpass $\left(E_{\text {iso }}^{\mathrm{BAT}}=(8.0 \pm 1.0) \times 10^{52} \mathrm{erg}\right.$, see Sect. 2.1). An upper limit to $E_{\text {iso }}$ is obtained in the most conservative case where the peak energy $E_{\mathrm{p}}$ is equal to $10^{4} \mathrm{keV}$ (Amati et al. 2002). By integrating the best fit BAT power-law spectrum in the whole $1-10^{4} \mathrm{keV}$ rest frame energy band we derived an upper limit of $4.5 \times 10^{53} \mathrm{erg}$ to $E_{\text {iso. }}$. Taking the central value of the interval above derived we obtained for GRB 050730 $E_{\text {iso }}=(2.6 \pm 1.9) \times 10^{53} \mathrm{erg}$.

With $z=3.969, t_{\mathrm{b}}=10.1_{-2.2}^{+4.6} \mathrm{ks}$ (see Sect. 3.1) and the $E_{\text {iso }}$ range above derived, we find $\theta_{\text {jet }}=1.6_{-0.2}^{+0.6} \mathrm{deg}$, for $\eta=0.2$ (Frail et al. 2001), assuming the value of circumburst density $n=10 \mathrm{~cm}^{-3}$ discussed by Bloom et al. (2003). With this value 
of the jet opening angle, the inferred collimation-corrected bolometric radiated energy is $E_{\text {jet }}=\left(1.0_{-0.8}^{+2.3}\right) \times 10^{50} \mathrm{erg}$.

Taking into account the $E_{\text {iso }}$ and $E_{\text {jet }}$ values derived above and the lower limit to the peak energy $\left(E_{\mathrm{p}}>750 \mathrm{keV}\right.$ rest frame), we find that GRB 050730 is consistent with the $E_{\mathrm{p}}$ vs. $E_{\text {iso }}$ relation found by Amati et al. (2002) and recently updated in Amati (2006). We also find that GRB 050730 is inconsistent, even taking into account the $3 \sigma$ scatter around the best fit correlation, with the $E_{\mathrm{p}}$ vs. $E_{\text {jet }}$ relation found by Ghirlanda et al. (2004) and with its updated version presented by Nava et al. (2006). In order to make this GRB consistent with the $E_{\mathrm{p}}-E_{\text {jet }}$ relation a much higher circumburst density $\left(n \sim 10^{5} \mathrm{~cm}^{-3}\right)$ would be required. GRB 050730 is inconsistent with the model-independent $E_{\text {iso }}-E_{\mathrm{p}}-t_{\mathrm{b}}$ correlation found by Liang \& Zhang (2005).

\section{Summary and conclusions}

We have presented a detailed temporal and spectral analysis of the afterglow of GRB 050730 observed with Swift and XMMNewton. The most striking feature of this GRB is the intense and exceptionally extended (over more than two orders of magnitude in time) X-ray flaring activity.

Superimposed to the afterglow decay we observed seven distinct re-brightening events peaking at $236 \mathrm{~s}, 437 \mathrm{~s}, 685 \mathrm{~s}, 4.5 \mathrm{ks}$, $10.4 \mathrm{ks}, 18.7 \mathrm{ks}$ and $41.2 \mathrm{ks}$ after the BAT trigger. The underlying decline of the afterglow was well described with a double broken power-law model with breaks at $t_{1}=237 \pm 20 \mathrm{~s}$ and $t_{2}=10.1_{-2.2}^{+4.6} \mathrm{ks}$. The temporal decay slopes before, between and after these breaks were $\alpha_{1}=2.1 \pm 0.3, \alpha_{2}=0.44_{-0.08}^{+0.14}$ and $\alpha_{3}=2.40_{-0.07}^{+0.09}$, respectively.

Strong spectral evolution during the flares was present together with an overall softening of the underlying afterglow with the energy index varying from $\beta=0.42 \pm 0.08$ during the early (133-205 s) steep decay to $\beta=0.99 \pm 0.05$ at much later $(50-60 \mathrm{ks})$ times. An absorbing column density $N_{\mathrm{H}}^{z}=$ $\left(1.28_{-0.25}^{+0.26}\right) \times 10^{22} \mathrm{~cm}^{-2}$ in the host galaxy is observed during the early (133-781 s) Swift observations while a lower column density $N_{\mathrm{H}}^{z}=(0.68 \pm 0.10) \times 10^{22} \mathrm{~cm}^{-2}$ is measured during the late (29.4-50.8 ks) XMM-Newton follow-up observation, likely indicating photo-ionization of the surrounding medium. Evidence of flaring activity in the early UVOT optical afterglow, simultaneous with that observed in the X-ray band, is found.

From the temporal analysis of the first three bright X-ray flares, we found that the rise and decay power-law slopes are in the range $0.8-1.8$ if the beginning and the peak of the flares are used as zero time, respectively. We also found that, with the exception of the first flare, for all episodes the ratio between the duration of the flare $(\Delta t)$ and the time when the flare peaks $\left(t_{\mathrm{p}}\right)$ is nearly constant and is $\Delta t / t_{\mathrm{p}} \sim 0.6-0.7$.

We showed that the observed properties of the first three flares are consistent with being due to both high-latitude emission, as expected for flares produced by late internal shocks, and to late time energy injection into the main afterglow shock by slow moving shells (refreshed shocks). An analysis of a larger sample of bursts would help in understanding what are the physical mechanisms responsible for the X-ray flaring activity.

We interpreted the X-ray temporal break at around $10 \mathrm{ks}$ as a jet break and derived a cone angle of $\sim 2$ deg and a radiated energy $E_{\text {jet }}=(0.2-3.3) \times 10^{50}$ erg against an isotropicequivalent energy $E_{\text {iso }}=(0.7-4.5) \times 10^{53} \mathrm{erg}$. GRB 050730 satisfies the $E_{\mathrm{p}}$ vs. $E_{\text {iso }}$ Amati relation but is inconsistent with the $E_{\mathrm{p}}$ vs. $E_{\text {jet }}$ Ghirlanda relation.
Acknowledgements. We are grateful to the referee for his/her useful comments and suggestions. We also thank C. Guidorzi for a very careful reading of the paper and F. Tamburelli and B. Saija for their work on the XRT data reduction software. This work is supported in Italy from ASI on contract number I/R/039/04 and through funding of the ASI Science Data Center, at Penn State by NASA contract NAS5-00136 and at the University of Leicester by the Particle Physics and Astronomy Research Council on grant numbers PPA/G/S/00524 and PPA/Z/S/2003/00507.

\section{References}

Amati, L. 2006, MNRAS, 372, 233

Amati, L., Frontera, F., \& Tavani, M. 2002, A\&A, 390, 81

Arnaud, K. A. 1996, Astronomical Data Analysis Software and Systems V, ed. Jacoby G. and Barnes J., ASP Conf. Ser., 101, 17

Band, D., Matteson, J., Ford, L., et al. 1993, ApJ, 413, 281

Barthelmy, S. D., Barbier, L. M.; Cummings, J. R., et al. 2005a, Space Sci. Rev., 120,143

Barthelmy, S. D., Cannizzo, J. K., Gehrels, N., et al. 2005b, ApJ, 635, L133

Bhatt, B. C., \& Sahu, D. K. 2005, GCN 3775

Bloom, J. S., Frail, D. A., \& Sari, S. R. 2001, AJ, 121, 2879

Bloom, J. S., Frail, D. A., \& Kulkarni, S. R. 2003, ApJ, 594, 674

Blustin, A. J., Holland, S. T., Cucchiara, N., et al. 2005, GCN 3717

Blustin, A. J., Band, D., Barthelmy, S., et al. 2006, ApJ, 637, 901

Burenin, R., Tkachenko, A., Pavlinsky, M., et al. 2005, GCN 3718

Burrows, D. N., Hill, J. E. Nousek, J. A., et al. 2005a, Space Sci. Rev., 120, 165

Burrows, D. N., Romano, P., Falcone, A., et al. 2005b, Science, 309, 1833

Cameron, P. B. 2005, GCN 3761

Campana, S., Beardmore, A. P., Cusumano, G., \& Godet. O. 2006, Swift-XRTCALDB-09 [http://swift.gsfc.nasa.gov/docs/heasarc/ caldb/swift/docs/xrt/SWIFT-XRT-CALDB-09.pdf]

Chen, H.-W., Prochaska, J. X., Bloom, J., \& Thompson, I. 2005a, ApJ, 634, L25

Chen, H.-W., Thompson, I., Prochaska, J. X., \& Bloom, J. 2005b, GCN 3709

Chincarini, G., Mangano, V., Moretti, A., et al. 2006, Proceedings of the conference The X-Ray Universe, San Lorenzo de El Escorial (Madrid, Spain), 26-30 September 2005 [arXiv: astro-ph/0511107]

Cobb, B. E., \& Bailyn, C. D. 2005, GCN 3708

Cusumano, G., Mangano, V., Angelini, L., et al. 2006a, ApJ, 639, 316

Cusumano, G., Mangano, V., Chincarini, G., et al. 2006b, Nature, 440, 164

Dai, Z. G., \& Cheng, 2001, ApJ, 558, L109

Damerdji, Y., Klotz, A., Boer, M., \& Atteia, J. L. 2005, GCN 3741

D'Elia, V., Melandri, A., Fiore, F., et al. 2005, GCN 3746

Dermer, C. 2004, ApJ, 614, 284

Dickey, J. M., \& Lockman., F. J. 1990, ARA\&A, 28, 215

Falcone, A. D., Burrows, D. N., Lazzati, D., et al. 2006, ApJ, 641, 1010

Fan, Y. Z., \& Wei, D. M. 2005, MNRAS, 364, L42

Frail, D. A., Kulkarni, S. R., Sari, R., et al. 2001, A\&A, 562, L55

Gehrels, N., Chincarini, G., Giommi, P., et al. 2004, ApJ, 611, 1005

Ghirlanda, G., Ghisellini, G., \& Lazzati, D. 2004, ApJ, 616, 331

Godet, O., Page, K. L., Osborne, J. P., et al. 2006, A\&A, 452, 819

Gomboc, A., Guidorzi, C., Steele, I. A., et al. 2005, GCN 3706

Granot, J., Nakar, E., \& Piran, T. 2003, Nature, 426, 138

Guetta, D., Fiore, F., D'Elia, V., et al. 2007, A\&A, 461, 95

Hill, J. E., Burrows, D. N., Nousek, J. A., et al. 2004, Proceedings of SPIE, 5165, 217

Holland, S. T., Barthelmy, S., Burrows, D. N., et al. 2005, GCN 3704

Holman, M., Garnavich, P., \& Stanek, K. Z. 2005a, GCN 3716

Holman, M., Garnavich, P., \& Stanek, K. Z. 2005b, GCN 3727

Hurkett, C. P., Osborne, J. P., Page, K. L., et al. 2006, MNRAS, 368, 1101

Ioka, K., Kobayashi, S., \& Zhang, B. 2005, ApJ, 631, 429

Jacques, C., \& Pimentel, E. 2005, GCN 3711

Kannappan, S., Garnavich, P., \& Stanek, K. Z. 2005, GCN 3778

King, A., O'Brien, P. T., Goad, M. R., et al. 2005, ApJ, 630, L11

Klotz, A., Boer, M., \& Atteia, J. L. 2005, GCN 3720

Kumar, P., \& Panaitescu, A. 2000, ApJ, 541, L51

Kumar, P., \& Piran, T. 2000, ApJ, 532, 286

Liang, E. W., \& Zhang, B., 2005, ApJ, 633, 611

Liang, E. W., Zhang, B., O'Brien, P. T., et al. 2006, ApJ, 646, 351

Mangano, V., et al. 2007, A\&A, submitted

Markwardt, C. B., Barbier, L., Barthelmy, S., et al. 2005, GCN 3715

Monfardini, L., Kobayashi, S., Guidorzi, C., et al. 2006, ApJ, 648, 1125

Moretti, A., Campana, S., Mineo, T., et al. 2005, Proc. SPIE, 5898, 360

Moretti, A., Perri, M., Capalbi, M., et al. 2006, A\&A, 448, L9

Nava, L., Ghisellini, G., Ghirlanda, G., Tavecchio, F., \& Firmani, C. 2006, A\&A, 450,471 
Nousek, J. A., Kouveliotou, C., Grupe, D., et al. 2006, ApJ, 642, 689 O'Brien, P.T., Willingale, R., Osborne, J., et al. 2006, ApJ, 647, 1213 Pagani, C., Morris, D. C., Kobayashi, S., et al. 2006, ApJ, 645, 1315

Pandey, S. B., Castro-Tirado, A. J., McBreen, S., et al. 2006, A\&A, 460, 415 Perna, R., Armitage, P. J., \& Zhang, B. 2006, ApJ, 636, L26

Perri, M., Giommi, P., Capalbi, M., et al. 2005, A\&A, 442 L1

Prochaska, J. X., Chen, H.-W., \& Bloom, J. 2006, ApJ, 648, 95

Rees, M. J., \& Mészáros, P. 1994, ApJ, 430, L93

Rees, M. J., \& Mészáros, P. 1998, ApJ, 496, L1

Rhoads, J. 1997, ApJ, 487, L1

Rol, E., Starling, R., Wiersema, K., et al. 2005, GCN 3710

Romano, P., Moretti, A., Banat, P. L., et al. 2006, A\&A, 450, 59
Roming, P. W. A., Kennedy, T. E., Mason, K. O., et al. 2005, Space Sci. Rev., 120, 95

Sari, R., \& Piran, T. 1997, ApJ, 485, 270

Sari, R., Piran, T., \& Narayan, N. 1998, ApJ, 497, L17

Sari, R., Piran, T., \& Halpern, J. P. 1999, ApJ, 519, L17

Sari, R., \& Mészáros, P. 2000, ApJ, 535, L33

Schlegel, D. J., Finkbeiner, D. P., \& Davis, M. 1998, ApJ, 500, 525

Sota, A., Castro-Tirado, A.J., Guziy, S., et al. 2005, GCN 3705

Starling, R. L. C., Vreeswijk, P. M., Ellison, S. L., et al. 2005, A\&A, 442, L21

Tagliaferri, G., Goad, M. R., Chincarini, G., et al. 2005, Nature, 436, 985

Vaughan, S., Goad, M. R., Beardmore, A. P., et al. 2006, ApJ, 638, 920

Zhang, B., Fan, Y. Z., Dyks, J., et al. 2006, ApJ, 642, 354 